\title{
Note on two extensions of the classical formula for sums of powers on arithmetic progressions
}

\author{
José A Adell ${ }^{*}$ and Alberto Lekuona
}

"Correspondence: adell@unizar.es Departamento de Métodos Estadísticos, Facultad de Ciencias, Universidad de Zaragoza, Pedro Cerbuna 12, Zaragoza, 50009, Spain

\begin{abstract}
We give two extensions of the classical formula for sums of powers on arithmetic progressions. This is achieved by using an identity involving binomial mixtures, which can be viewed as a generalization of the binomial transform.
\end{abstract}

MSC: Primary 05A19; secondary 60C05

Keywords: sum of powers formula; forward difference; binomial mixture; binomial transform; Bernoulli polynomials

\section{Introduction}

Let $\mathbb{N}$ be the set of nonnegative integers and $\mathbb{N}_{+}=\mathbb{N} \backslash\{0\}$. Throughout this note, we assume that $m, n \in \mathbb{N}, x \in \mathbb{R}$, and that $f: \mathbb{R} \rightarrow \mathbb{R}$ is an arbitrary function. The $k$ th forward differences of $f$ are recursively defined by $\Delta^{0} f(x)=f(x), \Delta^{1} f(x)=f(x+1)-f(x)$, and

$$
\Delta^{k} f(x)=\Delta^{1}\left(\Delta^{k-1} f\right)(x)=\sum_{i=0}^{k}\left(\begin{array}{l}
k \\
i
\end{array}\right)(-1)^{k-i} f(x+i), \quad k \in \mathbb{N}_{+} .
$$

The starting point of this note is the following classical formula for sums of powers:

$$
\sum_{k=0}^{n}(x+k)^{m}=\frac{B_{m+1}(x+n+1)-B_{m+1}(x)}{m+1}
$$

where $B_{m}(x)$ is the $m$ th Bernoulli polynomial. Since the time of James Bernoulli (1655$1705)$, several methods have been developed to find such sums, trying in many occasions to obtain different generalizations. For instance, Kannappan and Zhang [1] (see also the references therein) have used Cauchy's equation to prove (2), when the monomial function $x^{m}$ is replaced by a polynomial of degree $m$. Some $q$-analogues of formula (2) can be found in Guo and Zeng [2] and the references therein.

On the other hand, the sums in (2) can also be computed by means of the forward differences of the monomial function $\psi_{m}(u)=u^{m}, u \in \mathbb{R}$. We actually have (see, for instance,

\section{照 Springer}


Rosen [3], p.199, or Spivey [4])

$$
\sum_{k=0}^{n}(x+k)^{m}=\sum_{k=0}^{m \wedge n}\left(\begin{array}{l}
n+1 \\
k+1
\end{array}\right) \Delta^{k} \psi_{m}(x)
$$

where $m \wedge n=\min (m, n)$. For $x=0$, formula (3) can be written in terms of the Stirling numbers of the second kind $S(m, k)$ defined as

$$
S(m, k)=\frac{1}{k !} \Delta^{k} \psi_{m}(0)
$$

Computationally, formulas (2) and (3) are equivalent in the sense that the computation of a sum of $n+1$ terms is reduced to the computation of a polynomial in $n$ of degree $m+1$. However, (2) can easily be derived from (3) as follows. Suppose that $\left(P_{m}(x)\right)_{m \geq 0}$ is a sequence of polynomials satisfying

$$
\Delta^{1} P_{m+1}(x)=c_{m} \psi_{m}(x)
$$

for a certain constant $c_{m}$ only depending upon $m$. Then we have from (3), (4), and formula (7) below

$$
\begin{aligned}
\sum_{k=0}^{n}(x+k)^{n} & =\sum_{k=0}^{n}\left(\begin{array}{l}
n+1 \\
k+1
\end{array}\right) \Delta^{k} \psi_{m}(x) \\
& =\frac{1}{c_{m}} \sum_{k=0}^{n}\left(\begin{array}{l}
n+1 \\
k+1
\end{array}\right) \Delta^{k+1} P_{m+1}(x) \\
& =\frac{P_{m+1}(x+n+1)-P_{m+1}(x)}{c_{m}}
\end{aligned}
$$

The Bernoulli polynomials satisfy (4) with $c_{m}=m+1$. However, one can construct other sequences of polynomials $\left(P_{m}(x)\right)_{m \geq 0}$ fulfilling (4) (in this respect, see Luo et al. [5]). For this reason, we will extend formula (3) rather than (2). This is done in Theorem 2.1 below by means of a simple identity involving binomial mixtures.

\section{Main results}

Let $\mathbb{S}_{n}=\left(S_{n}(t), 0 \leq t \leq 1\right)$ be a stochastic process such that $S_{n}(t)$ has the binomial law with parameters $n$ and $t$, i.e.,

$$
P\left(S_{n}(t)=k\right)=\left(\begin{array}{l}
n \\
k
\end{array}\right) t^{k}(1-t)^{n-k}, \quad k=0,1, \ldots, n,
$$

and let $T$ be a random variable taking values in $[0,1]$ and independent of $\mathbb{S}_{n}$. The random variable $S_{n}(T)$, obtained by randomizing the success parameter $t$ by $T$, is called a binomial mixture with mixing random variable $T$ (see [6] and the references therein). As follows from (5), the probability law of $S_{n}(T)$ is given by

$$
P\left(S_{n}(T)=k\right)=\left(\begin{array}{l}
n \\
k
\end{array}\right) E\left[T^{k}(1-T)^{n-k}\right], \quad k=0,1, \ldots, n,
$$

where $E$ stands for mathematical expectation. Our first main result is the following. 
Theorem 2.1 With the preceding notations, we have

$$
E\left[f\left(x+S_{n}(T)\right)\right]=\sum_{k=0}^{n}\left(\begin{array}{l}
n \\
k
\end{array}\right) f(x+k) E\left[T^{k}(1-T)^{n-k}\right]=\sum_{k=0}^{n}\left(\begin{array}{l}
n \\
k
\end{array}\right) \Delta^{k} f(x) E\left[T^{k}\right] .
$$

Let $U$ be a random variable having the uniform distribution on $(0,1)$. Observe that

$$
E\left[U^{k}(1-U)^{n-k}\right]=\int_{0}^{1} \theta^{k}(1-\theta)^{n-k} d \theta=\beta(k+1, n-k+1)=\frac{k !(n-k) !}{(n+1) !},
$$

where $\beta(\cdot, \cdot)$ is Euler's beta function. Setting $T=U$ and $f=\psi_{m}$ in Theorem 2.1, we obtain (3), as follows from (6) and the fact that $\Delta^{k} \psi_{m}(x)=0, k=m+1, m+2, \ldots$ On the other hand, choosing $T=1$ in Theorem 2.1, we obtain the well-known identity (see, for instance, Flajolet and Vepstas [7])

$$
f(x+n)=\sum_{k=0}^{n}\left(\begin{array}{l}
n \\
k
\end{array}\right) \Delta^{k} f(x)
$$

In the terminology of binomial transforms (see, for instance, $\mathrm{Mu}$ [8] and the references therein), identity (7) means that $(f(x+k))_{k \geq 0}$ is the binomial transform of $\left(\Delta^{k} f(x)\right)_{k \geq 0}$. In this sense, Theorem 2.1 appears as a generalization of (7).

Every choice of the function $f$ and the random variable $T$ in Theorem 2.1 gives us a different binomial identity. Whenever the probability density of $T$ includes the uniform density on $(0,1)$ as a particular case, we are able to obtain a different extension of formula (3). In this respect, we give the following two corollaries of Theorem 2.1.

Corollary 2.2 For any $p>0$ and $q>0$, we have

$$
\sum_{k=0}^{n}\left(\begin{array}{c}
-p \\
k
\end{array}\right)\left(\begin{array}{c}
-q \\
n-k
\end{array}\right) f(x+k)=\sum_{k=0}^{n}\left(\begin{array}{c}
-p \\
k
\end{array}\right)\left(\begin{array}{c}
-(p+q+k) \\
n-k
\end{array}\right) \Delta^{k} f(x) .
$$

Finally, recall that the discrete Cesàro operator $C$ is defined as

$$
C f(x+n)=\frac{f(x)+f(x+1)+\cdots+f(x+n)}{n+1} .
$$

We denote by $C^{j}$ the $j$ iterate of $C, j \in \mathbb{N}_{+}$(see Galaz and Solís [9] and Adell and Lekuona [10] for the asymptotic behavior of such iterates, as $j \rightarrow \infty$ ).

Corollary 2.3 For any $j \in \mathbb{N}_{+}$, we have

$$
\begin{aligned}
C^{j} f(x+n) & =\sum_{k=0}^{n} f(x+k)\left(\begin{array}{l}
n \\
k
\end{array}\right) \int_{0}^{1} \theta^{k}(1-\theta)^{n-k} \frac{(-\log \theta)^{j-1}}{(j-1) !} d \theta \\
& =\sum_{k=0}^{n}\left(\begin{array}{l}
n \\
k
\end{array}\right) \frac{1}{(k+1)^{j}} \Delta^{k} f(x) .
\end{aligned}
$$

Observe that both corollaries extend formula (3) by choosing $f=\psi_{m}$ and $p=q=1$ in Corollary 2.2, and $f=\psi_{m}$ and $j=1$ in Corollary 2.3. 


\section{The proofs}

Proof of Theorem 2.1 Let $t \in[0,1]$. We have from (1) and (5)

$$
\begin{aligned}
E\left[f\left(x+S_{n}(t)\right)\right] & =\sum_{k=0}^{n}\left(\begin{array}{l}
n \\
k
\end{array}\right) f(x+k) t^{k}(1-t)^{n-k} \\
& =\sum_{k=0}^{n}\left(\begin{array}{l}
n \\
k
\end{array}\right) f(x+k) t^{k} \sum_{j=0}^{n-k}\left(\begin{array}{c}
n-k \\
j
\end{array}\right)(-t)^{j} \\
& =\sum_{s=0}^{n} \sum_{k+j=s}\left(\begin{array}{l}
n \\
k
\end{array}\right)\left(\begin{array}{c}
n-k \\
j
\end{array}\right)(-1)^{j} f(x+k) t^{k+j} \\
& =\sum_{s=0}^{n}\left(\begin{array}{l}
n \\
s
\end{array}\right) t^{s} \sum_{k=0}^{s}\left(\begin{array}{l}
s \\
k
\end{array}\right)(-1)^{s-k} f(x+k) \\
& =\sum_{s=0}^{n}\left(\begin{array}{l}
n \\
s
\end{array}\right) t^{s} \Delta^{s} f(x) .
\end{aligned}
$$

Thus, it suffices to replace $t$ by the random variable $T$ and then to take expectations.

Proof of Corollary 2.2 Let $T$ be a random variable having the beta density

$$
\rho(\theta)=\frac{\theta^{p-1}(1-\theta)^{q-1}}{\beta(p, q)}, \quad \theta \in(0,1), p>0, q>0 .
$$

As in (6), we have

$$
E\left[T^{r}(1-T)^{s}\right]=\frac{1}{\beta(p, q)} \int_{0}^{1} \theta^{p+r-1}(1-\theta)^{q+s-1} d \theta=\frac{\beta(p+r, q+s)}{\beta(p, q)},
$$

whenever $r>-p$ and $s>-q$. Hence, applying Theorem 2.1, we get

$$
\sum_{k=0}^{n}\left(\begin{array}{l}
n \\
k
\end{array}\right) \beta(p+k, q+n-k) f(x+k)=\sum_{k=0}^{n}\left(\begin{array}{l}
n \\
k
\end{array}\right) \beta(p+k, q) \Delta^{k} f(x) .
$$

The conclusion follows from (10) and the well-known formulas

$$
\beta(p, q)=\frac{\Gamma(p) \Gamma(q)}{\Gamma(p+q)}, \quad \frac{\Gamma(p+k)}{k ! \Gamma(p)}=(-1)^{k}\left(\begin{array}{c}
-p \\
k
\end{array}\right), \quad k \in \mathbb{N}
$$

Proof of Corollary 2.3 Let $j \in \mathbb{N}_{+}$. The following formula for the $j$ iterate of the discrete Cesàro operator $C$ was shown by Hardy [11], Section II.12,

$$
C^{j} f(x+n)=\sum_{k=0}^{n} f(x+k)\left(\begin{array}{l}
n \\
k
\end{array}\right) \int_{0}^{1} \theta^{k}(1-\theta)^{n-k} \frac{(-\log \theta)^{j-1}}{(j-1) !} d \theta
$$

A probabilistic representation of (11) can be built as follows (see [10] for more details). Let $\left(U_{k}\right)_{k \geq 1}$ be a sequence of independent identically distributed random variables having the 
uniform distribution on $(0,1)$, and denote $T_{j}=U_{1} \cdots U_{j}$. It turns out (cf. [10], Lemma 2.2) that the probability density of $T_{j}$ is given by

$$
\rho_{j}(\theta)=\frac{(-\log \theta)^{j-1}}{(j-1) !}, \quad 0<\theta<1 .
$$

On the other hand, we see that

$$
E\left[T_{j}^{k}\right]=E\left[U_{1}^{k}\right] \cdots E\left[U_{j}^{k}\right]=\frac{1}{(k+1)^{j}}, \quad k \in \mathbb{N} .
$$

Therefore, the conclusion follows by choosing $T=T_{j}$ in Theorem 2.1 and taking into account (11)-(13).

\section{Acknowledgements}

The authors would like to thank the reviewers for their careful reading of the manuscript and for their suggestions, which greatly improved the final outcome. This work was supported by research grants MTM2015-67006-P, DGA (E-64), and by FEDER funds.

\section{Competing interests}

The authors declare that they have no competing interests.

\section{Authors' contributions}

Both authors read and approved the final manuscript.

\section{Publisher's Note}

Springer Nature remains neutral with regard to jurisdictional claims in published maps and institutional affiliations.

Received: 3 March 2017 Accepted: 20 June 2017 Published online: 27 June 2017

\section{References}

1. Kannappan, PL, Zhang, W: Finding sum of powers on arithmetic progressions with application of Cauchy's equation. Results Math. 42(3-4), 277-288 (2002). doi:10.1007/BF03322855

2. Guo, VJW, Zeng, J: A q-analogue of Faulhaber's formula for sums of powers. Electron. J. Comb. 11(2), R19 (2005)

3. Rosen, KH: Handbook of Discrete and Combinatorial Mathematics. CRC Press, Boca Raton (2000)

4. Spivey, MZ: Combinatorial sums and finite differences. Discrete Math. 307(24), 3130-3146 (2007). doi:10.1016/j.disc.2007.03.052

5. Luo, Q-M, Qi, F, Debnath, L: Generalizations of Euler numbers and polynomials. Int. J. Math. Math. Sci. 61, 3893-3901 (2003). doi:10.1155/S016117120321108X

6. Adell, JA, Anoz, JM: Signed binomial approximation of binomial mixtures via differential calculus for linear operators. J. Stat. Plan. Inference 138(12), 3687-3695 (2008). doi:10.1016/j.jspi.2007.11.018

7. Flajolet, P, Vepstas, L: On differences of zeta values. J. Comput. Appl. Math. 220(1-2), 58-73 (2008). doi:10.1016/j.cam.2007.07.040

8. Mu, Y-P: Symmetric recurrence relations and binomial transforms. J. Number Theory 133(9), $3127-3137$ (2013). doi:10.1016/j.jnt.2013.03.003

9. Galaz Fontes, F, Solís, FJ: Iterating the Cesàro operators. Proc. Am. Math. Soc. 136(6), 2147-2153 (2008). doi:10.1090/S0002-9939-08-09197-1

10. Adell, JA, Lekuona, A: Rates of convergence for the iterates of Cesàro operators. Proc. Am. Math. Soc. 138(3), 1011-1021 (2010). doi:10.1090/S0002-9939-09-10127-2

11. Hardy, GH: Divergent Series. Clarendon, Oxford (1949) 